Gustavo Marins de Aguiar 2

Wagner Muniz de Medei ros 2

Tania Santos De Marco 2

Simone Corrêa dos Santos 2

Simone Gambardella 2

\section{Ecologia dos flebotomíneos da Serra do Mar, Itaguaí, Estado do Rio de J aneiro, Brasil. I - A fauna flebotomínica e prevalência pelo local e tipo de captura (Diptera, Psychodidae, Phlebotominae) 1}

\author{
Ecology of sandflies in Serra do Mar, Itaguaí, \\ state of Rio de J aneiro, Brazil. I - Sandfly fauna \\ and prevalence of the species in collections sites \\ and method of capture (Diptera, Psychodidae, \\ Phlebotominae) 1
}

${ }^{1}$ Trabal ho realizado com auxílio do CNPq

2 Departamento de Entomologia, Instituto Oswaldo Cruz, Fundação Oswaldo Cruz. Av. Brasil 4365 Rio de Janeiro, RJ, 21045-900, Brasil.
Abstract A two-year investigation was conducted in Itaguai, State of Rio de Janeiro, an area with cases of cutaneous leishmaniasis, in order to study the ecology of sandflies and their habits and role as parasitic vector for men and animals. Capturing took place at three sites: domiciliary (human bait, walls, and light traps); peridomi ciliary (walls, from baits used si multaneously: humans, dogs, and chickens; and light traps); and sylvatic (human bait and light traps). A total of 10,172 sandfl ies were captured, belonging to 17 species of genuses Brumptomyia França \& Parrot, 1921 and Lutzomyia França, 1924. L. intermedia was predominant, $100 \mathrm{~m}$ above sea level, as compared to L. migonei and L. fischeri. In a drier area $300 \mathrm{~m}$ above sea level L. migonei was prevalent, followed by L. longipalpis and L. fischeri. The species which presented the greatest endophily was L. fischeri, showing a certain degree of eclecti cism regarding the biting site. L. intermedia and L. migonei proved to be more exophilic. L. intermedia was considered to be potentially the main transmitter of the di sease because of its prevalence, anthropophily, and the fact it is known to be a vector of Leishmania (V.) braziliensis in other areas of the Southeastern region of Brazil. L. fischeri, because of its anthropophily, might also be involved.

Key words Lutzomyia; Sandflies; Epidemi ology; Ecology; Cutaneous Leishmaniasis

Resumo Durantedois anos foi feito um estudo ecológico sobre osflebotomíneos em foco de leishmani ose cutânea em Itaguaí, Estado do Rio de Janeiro. As capturas (isca humana, paredes e armadi lha lumi nosa) foram efetuadas, si multaneamente, em três síti os de col eta: domicílio, peridomicílio e floresta. Foram capturados 10.172 fl ebotomíneos, de 17 espécies, sendo 3 do gênero Brumptomyia e 14 do gênero Lutzomyia. A espécie mais preval ente a 100m do nível do mar éL. intermedia, seguida delonge por L. migonei eL. fischeri. A espécie mais endófila e que apresenta um certo ecl eti smo quanto ao local de hematofagia éL. fischeri, enquanto L. intermedia e L. migonei provaram ser mais exofílicas. L. intermedia pode ser incriminada como o principal vetor potencial do agente de leishmaniose tegumentar, pela sua prevalência, antropofilia e por ser comprovada a vei culação da Leishmania (Viannia) braziliensis em outras áreas do Estado do Rio de Janeiro. L. fischeri, pela avidez com que pica o homem, pode ser um coadjuvante na transmi ssão do parasita. Sua predominância na floresta sugere parti ci pação da transmissão em seu ciclo enzoótico natural. A presença de L. Iongipal pis é um risco potencial de vei culação do agente etiológico da leishmaniose visceral nessa região, particularmente pela baixa imunidade da população local.

Palavras-chave Lutzomyia; Flebotomíneos; Epidemiologia; Ecologia; Leishmaniose Tegumentar 


\section{Introdução}

O estudo da ecologia dos flebotomíneos no Estado do Rio de Janeiro foi iniciado quando da ocorrência de casos de leishmaniose tegumentar na antiga capital da República. Desde então, a parasitose vem ocorrendo de forma endêmica, epidêmica ou de casos esporádicos. No entanto, os pesquisadores centralizavam suas investigações, principalmente, nos aspectos clínicos e terapêuticos da doença. A partir da década de 1940, o uso de inseticidas como medida profilática, nas casas e nos anexos de animais domésticos, dificultou o estudo dos hábitos dos vetores e sua relação na transmissão do parasita ao homem e animais. Mesmo assim, foi notório o progresso nas investigações de campo e laboratório. As informações disponíveis são, no entanto, ainda insuficientes para se conhecer mel hor a relação dos vetores com a doença. Atualmente se considera a Leishmania (Viannia) braziliensiso principal agente etiológico da leishmaniose tegumentar no Rio de Janeiro, sendo a infecção de animais domésticos, como cães e eqüídeos, associada a flebotomíneos que se adaptam bem aos ambientes modificados, os quais propiciam aos insetos abrigo e alimento em maior abundância que nos ambientes naturais (Marzochi \& Marzochi, 1994). A ocorrência recente de inúmeros casos de leishmaniose tegumentar no Município de Itaguaí e, principalmente, a constatação da ocorrência de casos da doença no sítio Porangaba, além da escassez de estudos sobre o assunto na área, ensejou a realização de um estudo prolongado e sistematizado sobre a ecologia dos flebotomíneos e o papel desses na transmissão do parasita ao homem e outros mamíferos.

Área de estudo

O Município de Itaguaí, Estado do Rio de Janeiro, está localizado entre as coordenadas de $22^{\circ}$ $42^{\prime}$ de latitude Sul e de $43^{\circ} 41^{\prime}$ de longitude Oeste, na zona fisiográfica da baixada do rio Guandu, limitando-se com os Municípios de Rio Claro, Piraí, Paracambi, Nova I guaçu, Mangaratiba e Rio de Janeiro. A região destaca-se como a principal produtora de banana do Estado, já que o clima quente e úmido Ihe é favorável. A cultura é feita preferencialmente nos grotões das encostas da Serra do Mar, alongando-se até os Municípios de Nova I guaçu e Duque de Caxias, RJ (FUNDREN, 1976).

O fracionamento das propriedades por empresas de loteamento extravasa as áreas que lhe são periféricas, valorizando as terras ao longo ou próximas das vias de comunicação. Em con- trapartida, imensas glebas permanecem desocupadas aguardando maior valorização.

Com todo este processo de ocupação, houve uma diminuição acentuada da fauna e da flora regionais, que, se antes eram abundantes e diversificadas, agora estão restritas às matas residuais remanescentes da Floresta Pereniforme Higrófila Costeira (Alonso, 1977) e limitadas às raras áreas de preservação como o Horto Florestal Municipal e outras propriedades particulares, onde se inclui o sítio Porangaba.

A área estudada situa-se nas proximidades da Fazenda São Sebastião, Raiz da Serra, Estado do Rio de Janeiro, com acesso pela rodovia municipal IG-06 a partir do centro do Município, que é interligado à rodovia Rio-Santos, BR 101. A sede do sítio fica a $100 \mathrm{~m}$ do nível do mar ( Figura 1).

\section{$M$ ateriais e métodos}

Para o presente estudo era necessário que a área não sofresse ação de inseticida usado pela Fundação Nacional de Saúde durante o desenvolvimento do trabal ho. Neste aspecto, foi decisiva a compreensão do proprietário do sítio que, interessando-se pela pesquisa, não só estabeleceu contato permanente com o Laboratório de Diptera, setor Phlebotominae, na Fundação Oswaldo Cruz, como também cedeu algumas instalações, facilitando, assim, a rotina da equipe durante a permanência na área.

Durante 24 meses, realizaram-se capturas mensais de flebotomíneos, com permanência de dois dias consecutivos na área. Em todas as capturas utilizou-se o tubo de sucção manual. As armadilhas usadas foram a luminosa, modelo Falcão (Falcão, 1981), modificada por Aguiar et al. (1985 a); a de Disney (Disney, 1966) e a de Damasceno (Damasceno, 1955).

A primeira estação de captura, situada a $100 \mathrm{~m}$ do nível do mar (Estação A), foi instalada na casa que serve de moradia aos caseiros do sítio. O local écircundado por plantação de banana, que se extende por um raio de $300 \mathrm{~m}$ até a floresta. A segunda estação foi fixada a $300 \mathrm{~m}$ do nível do mar (Estação B), ainda nos limites da propriedade. No local, prevalecem as plantações de milho e de mandioca em área mais seca e sem a presença do bananal. A floresta dista, aproximadamente, $450 \mathrm{~m}$ da estação.

A escol ha das casas como pontos referenciais para as capturas de flebotomíneos deveuse à constatação de casos de leishmaniose tegumentar em indivíduos do mesmo domicílio e a nítida diferença entre as duas áreas, que, embora relativamente próximas, apresentam 

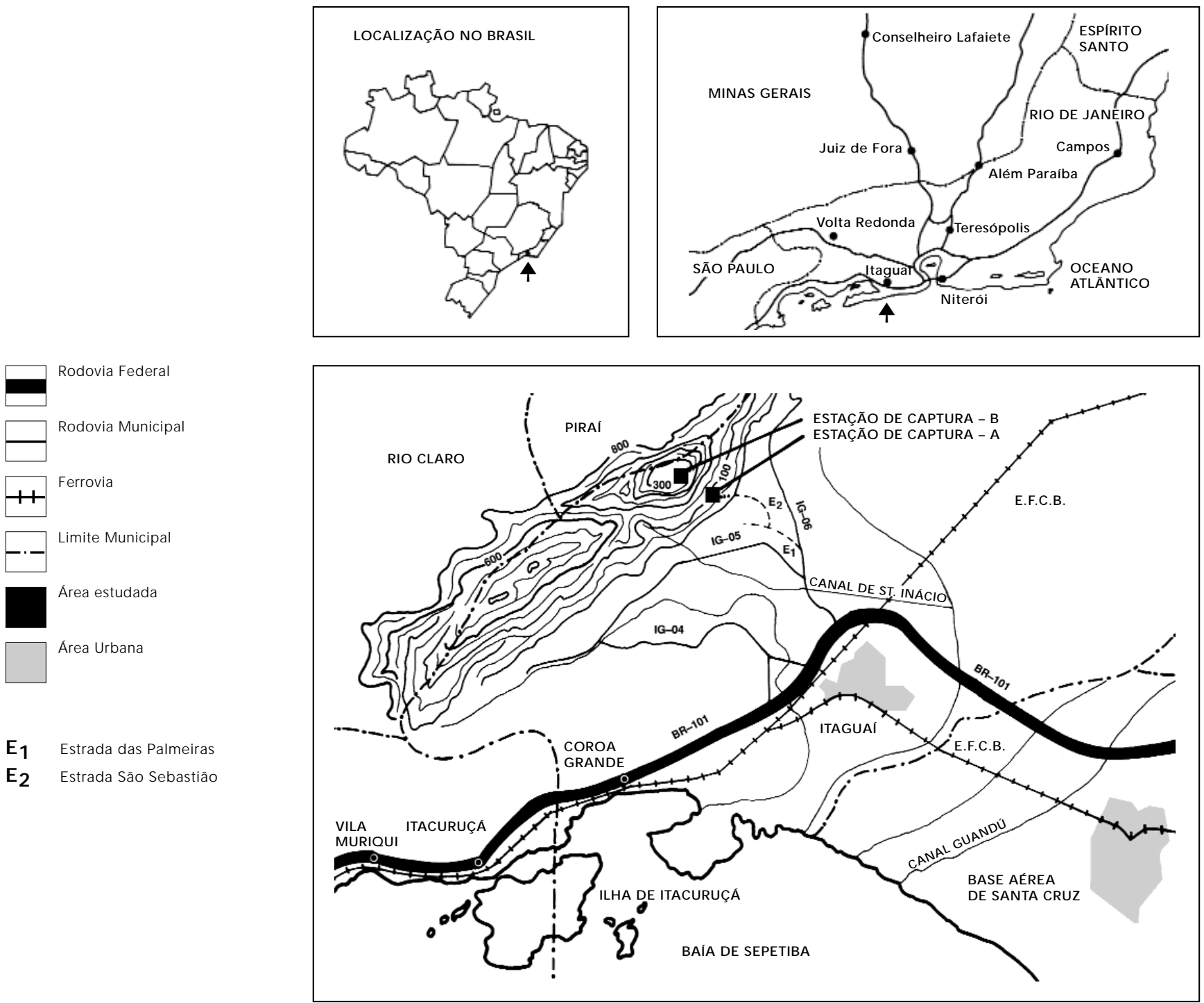

E1 Estrada das Palmeiras

E2 Estrada São Sebastião

características diferentes, como mencionado anteriormente.

\section{Programação das capturas}

De março de 1984 a fevereiro de 1985, na Estação A, e de março de 1985 a fevereiro de 1986, na Estação B, realizaram-se capturas mensais de flebotomíneos. Elas foram efetuadas, simultaneamente, em três sítios de coleta: domicílio (isca humana e paredes internas), peridomicílio (isca humana e paredes externas) efloresta (isca humana), nos seguintes períodos - matutino, das $6 \mathrm{~h}$ às $8 \mathrm{~h}$; vespertino, das $17 \mathrm{~h}$ às $19 \mathrm{~h} \mathrm{e}$ noturno, das $20 \mathrm{~h}$ às $22 \mathrm{~h}$ e de $0 \mathrm{~h}$ às $2 \mathrm{~h}$.
As armadilhas luminosas, em número de três, foram utilizadas nos dois anos, nas estações A e B, no interior da casa, no peridomicílio (próximo aos anexos de animais domésticos) e na floresta, sempre no mesmo local e hora, ou seja, eram ligadas às $18 \mathrm{~h}$ e desligadas às 6h da manhã seguinte.

Como o objetivo era capturar flebotomíneos de vôo baixo e especialmente aqueles atraídos pelo sangue de roedores, foram utilizados dois desses animais - Akodon sp. e Oxymycterus sp. - nas armadilhas Disney. O primeiro foi capturado e usado como isca no peridomicílio, o mesmo ocorrendo com o segundo no interior do bananal. As armadilhas foram insta- 
ladas a $20 \mathrm{~cm}$ do solo, em locais previamente selecionados do peridomicílio e do bananal, das $18 \mathrm{~h}$ às $10 \mathrm{~h}$ da manhã seguinte.

A armadilha Damasceno foi empregada apenas onde os flebotomíneos ficam confinados, como as tocas de tatu, buracos no solo e ocos de árvores.

A maioria dos flebotomíneos capturados em isca humana foi levada viva, em pequenas caixas de polietileno, com fina camada de gesso, para ser dissecada. As caixas eram colocadas dentro de um isopor que, forrado com uma toalha umidecida, permitia a sobrevivência desses insetos. No laboratório, eram mergulhados em soro fisiológico e transferidos, individualmente, para uma lâmina com uma gota de soro, onde a cabeça era removida e o tubo digestivo levemente retirado pela parte posterior do abdômen. Após este procedimento, a lâmina era transferida ao microscópio para diagnóstico da espécie, procura de flagelados e sua localização no tubo digestivo. A identificação das espécies foi feita pelas espermatecas no momento da dissecção. Os exemplares machos e as fêmeas não destinadas à procura de flagelados foram transportados em pequenos tubos contendo álcool a $70^{\circ}$, com os respectivos rótulos da captura.

\section{Resultados}

Foram capturados 10.172 flebotomíneos, pertencentes às dezessete espécies listadas a seguir, com a designação de Martins et al. (1978):

Brumptomyia avellari (Costa Lima, 1932) Brumptomyia cunhai (Mangabeira, 1942) Brumptomyia guimaraesi (Coutinho \& Barretto, 1941) Lutzomyia (Lutzomyia) longipalpis (Lutz \& Neiva, 1912)

Lutzomyia (Pintomyia ) fischeri (Pinto, 1926)

Lutzomyia (Pintomyia) pessoai (Coutinho \&

Barretto, 1940)

Lutzomyia (Pintomyia) sp.

Lutzomyia (Micropygomyia) schreiberi

(Martins, Falcão \& Silva, 1975)

Lutzomyia (Helcocyrtomyia) quinquefer

(Dyar, 1929)

Lutzomyia (Nyssomyia) intermedia (Lutz \& Neiva, 1912)

Lutzomyia (Nyssomyia) whitmani (Antunes

$\&$ Coutinho, 1939)

Lutzomyia edwardsi (Mangabeira, 1941)

Lutzomyia migonei (França ,1920)

Lutzomyia barrettoi (Mangabeira, 1941)

Lutzomyia lanei (Barretto \& Coutinho, 1941)
Lutzomyia firmatoi (Barretto, Martins \&

Pellegrino, 1956)

Lutzomyia monticola (Costa Lima, 1932)

Do gênero Brumptomyia (França \& Parrot, 1921), foram capturados 82 exemplares de três espécies; de Lutzomyia (França, 1924), 10.091 exemplares pertencentes a catorze espécies. Lutzomyia (Pintomyia) sp. não teve a sua identificação confirmada até o momento. As demais já haviam sido assinaladas no Estado do Rio de Janeiro.

Os flebotomíneos identificados pertencem a cinco subgêneros: Lutzomyia, Helcocyrtomyia, Pintomyia, Micropygomyia e Nyssomyia, três grupos de espécies - migonei, brasiliensise Ianei - e duas espécies isoladas, L. firmatoi e L. monticola.

Na Tabela 1, representam-se o número e a média, por 10h, de flebotomíneos capturados em isca humana (domicílio, peridomicílio e floresta) e pousados nas paredes (interna e externa) do domicílio, a 100 e 300m do nível do mar. Tanto no primeiro como no segundo nível foram gastas $288 \mathrm{~h}$ de captura, somando-se os três sítios de coleta. As médias obtidas a 100m foram sempre superiores às de $300 \mathrm{~m}$, em decorrência da ampla prevalência de L. intermedia sobre L. migonei e L. fischeri. Entre as menos numerosas, destacam-se L. schreiberi, L. firmatoi e L. monticola. A 300m, o predomínio é de L. migonei, com ampla vantagem sobre L. fischeri e L. longi pal pis. Das espécies menos comuns, registram-se as presenças de L. whitmani, única capturada somente neste nível, e L. intermedia.

Nas Figuras 2 e 3, comparam-se o total e a freqüência das espécies mais numerosas a 100 e 300m do nível do mar. Com relação ao total, em ambos os níveis, nota-se que a maioria dos flebotomíneos ocorre nas paredes externas e internas do domicílio. No primeiro nível, o homem é mais picado no peridomicílio, ao passo que, no segundo, no domicílio. Em relação às espécies capturadas em isca humana, demonstra-se que L. intermedia tem grande prevalência no peridomicílio e em menor proporção no domicílio; na floresta não foi encontrada. L. migonei mostra a mesma tendência, no entanto, aparece com maior equilíbrio; na floresta sua presença é insignificante. L. fischeri, ao contrário das anteriores, é mais freqüente no domicílio, evidenciando-se também números expressivos no peridomicílio e na floresta, sendo neste local a espécie predominante. No segundo nível, L. migonei e L. fischeri ocorrem em maior número no domicílio, enquanto L. longipalpis prevalece no peridomicílio, não 
Total e média, por $10 \mathrm{~h}$ de captura, de flebotomíneos, fêmeas e machos, capturados simultaneamente em isca humana (domicílio, peridomicílio e floresta) e pousados nas paredes (internas e externas), a 100 e $300 \mathrm{~m}$ do nível do mar, de março de 1984 a fevereiro de 1985 e de março de 1985 a fevereiro de 1986, Itaguaí, Estado do Rio de J aneiro.

\begin{tabular}{|c|c|c|c|c|c|c|c|c|c|c|}
\hline \multirow[t]{3}{*}{ Nível } & \multirow[t]{3}{*}{ Espécie } & \multicolumn{4}{|c|}{ Domicílio } & \multicolumn{4}{|c|}{ Peridomicílio } & \multirow{3}{*}{$\begin{array}{c}\text { Floresta } \\
\text { IH } \\
\text { F }\end{array}$} \\
\hline & & \multirow{2}{*}{$\begin{array}{c}\mathrm{IH} \\
\mathrm{F}\end{array}$} & \multicolumn{3}{|c|}{ Paredes } & \multirow{2}{*}{$\begin{array}{c}\mathrm{IH} \\
\mathrm{F}\end{array}$} & \multicolumn{3}{|c|}{ Paredes } & \\
\hline & & & $\mathrm{T}$ & $\mathrm{F}$ & $M$ & & $\mathrm{~T}$ & $\mathrm{~F}$ & M & \\
\hline \multirow[t]{9}{*}{$100 m$} & L. intermedia & 88 & 870 & 393 & 477 & 329 & 2687 & 1020 & 1667 & - \\
\hline & L. migonei & 42 & 257 & 97 & 160 & 66 & 518 & 145 & 373 & 3 \\
\hline & L. fischeri & 127 & 286 & 286 & - & 90 & 119 & 119 & - & 68 \\
\hline & L. schreiberi & - & 1 & 1 & - & - & 22 & 16 & 6 & 4 \\
\hline & L. firmatoi & - & - & - & - & - & 1 & 1 & - & 7 \\
\hline & L. monticola & - & - & - & - & - & - & - & - & 7 \\
\hline & $* 0$ utras & - & 1 & 1 & - & 1 & 2 & 2 & - & 2 \\
\hline & Total & 257 & 1415 & 778 & 637 & 486 & 3349 & 1303 & 2046 & 91 \\
\hline & Horas gastas & 96 & 96 & & & 96 & 96 & & & 96 \\
\hline \multirow[t]{7}{*}{$300 m$} & L. migonei & 45 & 266 & 91 & 175 & 43 & 209 & 37 & 172 & 2 \\
\hline & L. fischeri & 51 & 66 & 66 & - & 33 & 68 & 53 & 15 & 12 \\
\hline & L. longipalpis & 5 & 42 & 8 & 34 & 5 & 176 & 24 & 152 & - \\
\hline & L. whitmani & - & - & - & - & 4 & 20 & 11 & 9 & 3 \\
\hline & $* 0$ utras & - & - & - & - & - & 16 & 13 & 3 & 14 \\
\hline & Total & 101 & 374 & 165 & 209 & 85 & 489 & 138 & 351 & 31 \\
\hline & Horas gastas & 96 & 96 & & & 96 & 96 & & & 96 \\
\hline \multirow[t]{8}{*}{$100 m$} & L. intermedia & 9,1 & 90,6 & 40,9 & 49,6 & 34,2 & 279,8 & 106,2 & 173,6 & - \\
\hline & L. migonei & 4,3 & 26,7 & 10,1 & 16,6 & 6,8 & 53,9 & 15,1 & 38,8 & 0,3 \\
\hline & L. fischeri & 13,2 & 29,7 & 29,7 & - & 9,3 & 12,3 & 12,3 & - & 7,0 \\
\hline & L. schreiberi & - & 0,1 & 0,1 & - & - & 2,2 & 1,6 & 0,6 & 0,4 \\
\hline & L. firmatoi & - & - & - & - & - & 0,1 & 0,1 & - & 0,7 \\
\hline & L. monticola & - & - & - & - & - & - & - & - & 0,7 \\
\hline & *O utras & - & 0,1 & 0,1 & - & 0,1 & 0,2 & 0,2 & - & 0,2 \\
\hline & Total & 26,7 & 147,3 & 81,0 & 66,3 & 50,6 & 348,8 & 135,7 & 213,1 & 9,4 \\
\hline \multirow[t]{6}{*}{$300 m$} & L. migonei & 4,6 & 27,7 & 9,4 & 18,2 & 4,4 & 21,7 & 3,8 & 17,9 & 0,2 \\
\hline & L. fischeri & 5,3 & 6,8 & 6,8 & - & 3,4 & 7,0 & 5,5 & 1,5 & 1,2 \\
\hline & L. longipalpis & 0,5 & 4,3 & 0,8 & 3,5 & 0,5 & 18,3 & 2,5 & 15,8 & - \\
\hline & L. whitmani & - & - & - & - & 0,4 & 2,0 & 1,1 & 0,9 & 0,3 \\
\hline & *O utras & - & - & - & - & - & 1,6 & 1,3 & 0,3 & 1,4 \\
\hline & Total & 10,5 & 38,9 & 17,1 & 21,7 & 8,8 & 50,9 & 14,3 & 36,5 & 3,2 \\
\hline
\end{tabular}

T - total; F - fêmea; M - macho; IH - isca humana; L - Lutzomyia; B - Brumptomyia

*O utras espécies: 100m - L. edwardsi, L. pessoai, L. Iongipalpis e L. sp. 300m - B. guimaraesi, B. avellari, B. cunhai,

L. firmatoi, L. edwardsi, L. barrettoi, L. monticola, L. schreiberi, L. lanei, L. intermedia, L. pessoai, L. quinquefer e L. sp.

ocorrendo na floresta. Em isca humana, observa-se um pequeno predomínio de L. fischeri sobre L. migonei no domicílio; contudo, no peridomicílio ocorre o inverso. L. longipalpis demonstra baixa antropofilia, picando o homem, na mesma proporção, dentro e fora da casa.

A Tabela 2 indica o número e a média, por 10h, de flebotomíneos capturados em armadiIhas Iuminosas instaladas no domicílio, peridomicílio e na floresta, a 100 e 300m do nível do mar. Em ambos os níveis, foram gastas 432h; tal como as capturas realizadas em isca humana e paredes, as armadilhas luminosas tenderam a apresentar médias mais elevadas no peridomicílio e no domicílio, ocorrendo maior número de espécies na floresta. L. fischeri, a $100 \mathrm{~m}$, foi numericamente dominante sobre $\mathrm{L}$. edwardsi e L. firmatoi, porém, a 300m do nível do mar, a situação se inverte. Com relação ao sexo, analisando-se os dois níveis, observa-se um certo equilíbrio. L. intermedia, L. migonei e L. Iongi pal pis tiveram um predomínio de machos no domicílio e no peridomicílio, enquanto L. fischeri foi capturada com supremacia quase absoluta de fêmeas, nos três locais onde foram expostas as armadilhas.

Na Tabela 3, demonstram-se o número e as médias, por 10h, de flebotomíneos, fêmeas e machos, capturados no peridomicílio e no bananal, utilizando-se armadilha Disney, com isca roedor, a 100m do nível do mar. Em 384h foram obtidos apenas 88 exemplares, sendo 26 no peridomicílio e 62 no bananal. Cinco espécies foram atraídas pelos roedores: L. firmatoi, L. intermedia e L. schreiberi, mais freqüentes no bananal, enquanto L. migonei e L. fischeri, 
Total e freqüência das espécies Lutzomyia intermedia, L. migonei e L. fischeri, em capturas simultâneas realizadas nas paredes (interna e externa) e em isca humana (domicílio, peridomicílio e floresta), a 100m do nível do mar, de março de 1984 a fevereiro de 1985, Itaguaí, Estado do Rio de J aneiro.

Total

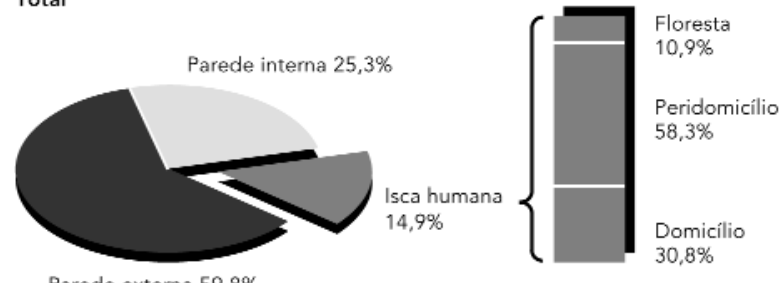

Parede externa $59,8 \%$

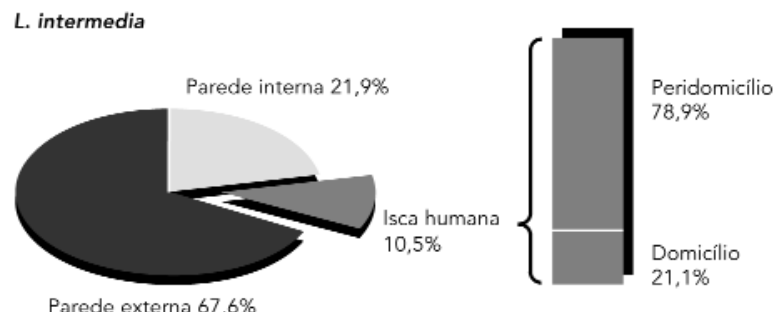

Parede externa $67,6 \%$

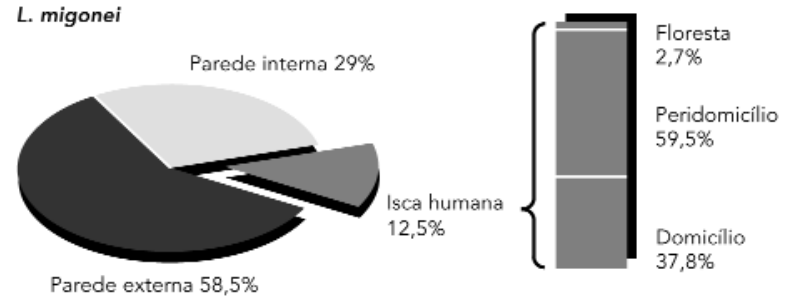

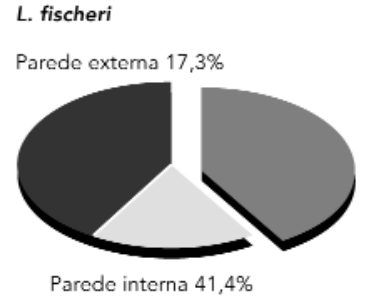

Floresta

$23,8 \%$

Peridomicílio $31,6 \%$ Isca humana $41,3 \%$

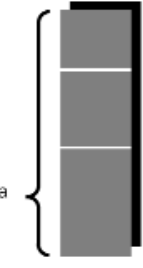

Domicilio $44,6 \%$

no peridomicílio, sendo a última capturada somente neste local. Com relação ao sexo, o número total de fêmeas supera o de machos e apenas L. migonei tem número de machos pouco mais elevado no peridomicílio.

Na Tabela 4, representa-se o número de flebotomíneos capturados em abrigos naturais, utilizando-se armadilha Damasceno, a 100m do nível do mar. Em 72h, foram obtidos 144 exemplares, pertencentes a oito espécies. A maioria dos flebotomíneos ocorreu em toca de tatu, sendo B. guimaraesi a mais numerosa, seguida por L. barrettoi, L. edwardsi e B. avellari. Em tronco de bananeira, foram capturadas $L$. firmatoi e L. intermedia. Em oco de árvore, L. fischeri foi mais freqüente que L. monticola; entretanto, em tronco de árvore, essa espécie foi a única capturada. Excetuando-se L. fischeri e L. monticola, evidencia-se um predomínio de machos.

Para a observação de infecção natural, foram dissecadas 1.232 fêmeas. Todas apresentaram resultados negativos. De L. intermedia, foram 582 exemplares; de L. migonei, 362; de L. fischeri, 234 e de L. Iongipal pis, 54.

\section{Discussão}

Durante o processo de colonização das regiões Sudeste e Sul, nas décadas de 30 e 40, a veiculação do agente etiológico da leishmaniose tegumentar esteve associada a L. whitmani, L. pessoai e L. mi gonei, espécies de comportamento silvestre. Atualmente, naquelas mesmas regiões, L. intermedia preval ece nas áreas endêmicas litorâneas e serranas dos Estados do Espírito Santo (Falqueto et al., 1986), Rio de Janeiro, no litoral, capital e interior (Aguiar et al., 1987, 1993; Souza et al., 1981) e São Paulo, onde o flebotomíneo é encontrado nos vales dos grandes rios (Gomes \& Galati, 1989; Tolezano et al., 1980). Nos Estados de Minas Gerais e Bahia, em áreas do interior (Três Braços), o flebotomíneo incriminado é L. whitmani (Mayrink et al., 1979; Vexenat \& Barreto, 1986a). No Estado do Ceará (Serra do Baturité), em ambiente florestal, o vetor é L. wellcomei (Ready et al., 1983), embora a principal forma de transmissão seja a periurbana e esteja associada a L. whitmani eL. migonei. No norte do Estado do Paraná, L. intermedia predomina no peridomicílio, L. whitmani, na margem da floresta e L. fischeri, no interior da mesma (Aguiar et al., 1989). 
Total e freqüência das espécies Lutzomyia migonei, L. fischeri e L. longipalpis, em capturas simultâneas realizadas nas paredes (interna e externa) e em isca humana (domicílio, peridomicílio e floresta), a 300m do nível do mar, de março de 1985 a fevereiro de 1986, Itaguaí, Estado do Rio de J aneiro.

Total

Parede externa $45,3 \%$

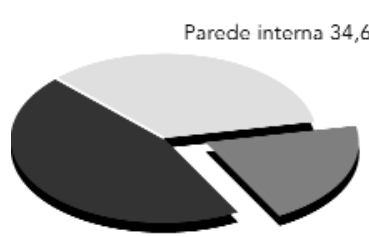

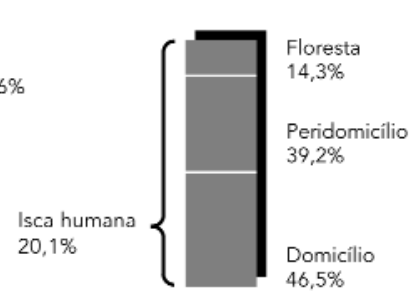

$46,5 \%$

Floresta

Peridomicílio $39,2 \%$

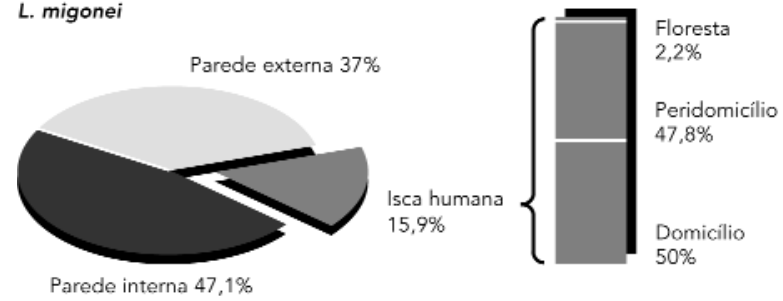

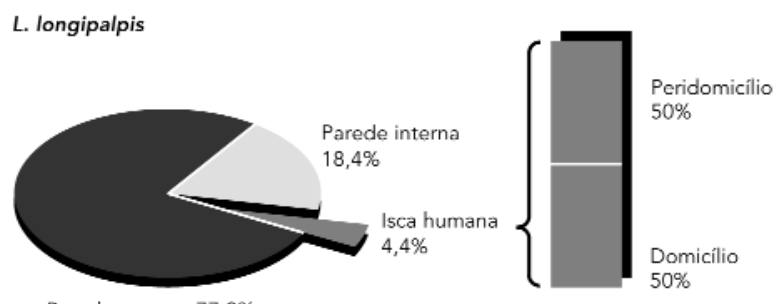

Parede externa $77,2 \%$

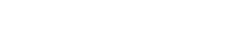
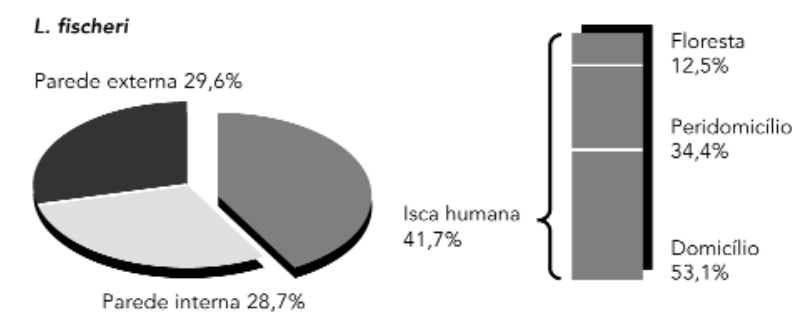

Nos primeiros estudos sobre a leishmaniose tegumentar no Brasil já se evidenciava a presença de L. intermedia em ambiente modificado, porém, somente a partir das pesquisas realizadas por Forattini \& Santos (1952) é que foi constatada uma alta densidade desse flebotomíneo dividindo a fauna alternadamente com L. whitmani no Estado de São Paulo. Posteriormente, veio a hipótese de sua participação como transmissor principal da Leishmania braziliensis (Forattini \& Oliveira, 1957).

A capacidade adaptativa de L. intermedia aos ecótopos artificiais, observada por Gomes et al. (1980), fortaleceu a hipótese levantada por Forattini et al. (1976) de incriminar a espécie como principal vetor do parasita em áreas periurbanas.

Ao longo dos anos, em Itaguaí, a crescente devastação da floresta e a expansão cafeeira, posteriormente substituída pela cultura de banana, onde os habitantes freqüentemente constroem suas casas, hábito iniciado pelos loteamentos irregulares na década de 50 , ocasionou a diminuição da fauna flebotomínica e a de animais si lvestres, porém elevou a densidade de L. intermedia no ambiente peridomiciliar e domiciliar, onde a espécie encontrou sua sobrevi- vência garantida graças à estreita relação alimentar com o homem, animais domésticos e sinantrópicos.

Analisando-se a fauna flebotomínica de Itaguaí, Estado do Rio de Janeiro, verifica-se que L. intermedi a é a espécie predominante nas capturas a 100 m do nível do mar, enquanto L. migonei prevalece a $300 \mathrm{~m}$, em área mais seca e sem a presença do bananal. L. Iongipal pis neste nível, foi a segunda em ordem de freqüência. Tal constatação já havia sido feita por Souza et al. (1981), no subúrbio de Bangu, na cidade do Rio de Janeiro. Por falta de investigações anteriores na área estudada, não foi possível determinar se a colonização de L. Iongi palpis é recente. Quanto a L. fischeri, em ambos os níveis, esteve bem representada, sendo a tercei ra pela ordem de freqüência, o que indica um certo grau de adaptação ao ambiente peridomiciliar e, especialmente, domiciliar. Estas observações são contrastantes com as de Barretto (1943) em São Paulo e Araújo Filho (1978) na Il ha Grande, Estado do Rio de Janeiro; entretanto, são concordantes com as investigações de Mattos (1981) em Perobas, no Estado do Espírito Santo e de Aguiar et al. (1991, 1993) em Picinguaba e Paraty, respectivamente nos Estados de São Paulo e Rio de Janeiro. 
Total e média, por 10h de captura, de flebotomíneos, fêmeas e machos, obtidos em armadilhas luminosas instaladas no domicílio, peridomicílio e floresta, a 100 e 300m do nível do mar, de março de 1984 a fevereiro de 1985 e de março de 1985 a fevereiro de 1986, Itaguaí, Estado do Rio de J aneiro.

\begin{tabular}{|c|c|c|c|c|c|c|c|c|c|c|}
\hline \multirow[t]{2}{*}{ Nível } & \multirow[t]{2}{*}{ Espécie } & \multicolumn{3}{|c|}{ Domicílio } & \multicolumn{3}{|c|}{ Peridomicílio } & \multicolumn{3}{|c|}{ Floresta } \\
\hline & & $\mathrm{T}$ & $\mathrm{F}$ & $M$ & $\mathrm{~T}$ & $\mathrm{~F}$ & M & $\mathrm{T}$ & $\mathrm{F}$ & $M$ \\
\hline \multirow[t]{9}{*}{$100 \mathrm{~m}$} & L. intermedia & 104 & 23 & 81 & 1555 & 722 & 833 & - & - & - \\
\hline & L. migonei & 51 & 18 & 33 & 605 & 214 & 391 & 1 & - & 1 \\
\hline & L. fischeri & 49 & 49 & - & 194 & 194 & - & 64 & 64 & - \\
\hline & L. edwardsi & - & - & - & 5 & 2 & 3 & 17 & 13 & 4 \\
\hline & L. firmatoi & - & - & - & - & - & - & 19 & 14 & 5 \\
\hline & L. schreiberi & - & - & - & 1 & 1 & - & 15 & 6 & 9 \\
\hline & $* 0$ utras & - & - & - & 6 & 5 & 1 & 17 & 8 & 9 \\
\hline & Total & 204 & 90 & 114 & 2366 & 1138 & 1228 & 133 & 105 & 28 \\
\hline & Horas Gastas & 144 & 144 & 144 & & & & & & \\
\hline \multirow[t]{11}{*}{$300 m$} & L. migonei & 35 & 6 & 29 & 147 & 51 & 96 & 3 & 2 & 1 \\
\hline & L. longipalpis & 16 & 5 & 11 & 90 & 36 & 54 & - & - & - \\
\hline & L. firmatoi & - & - & - & 6 & 4 & 2 & 69 & 57 & 12 \\
\hline & L. edwardsi & - & - & - & 3 & 3 & - & 59 & 21 & 38 \\
\hline & L. fischeri & 11 & 11 & - & 31 & 29 & 2 & 15 & 15 & - \\
\hline & L. whitmani & - & - & - & 9 & 9 & - & 8 & 8 & - \\
\hline & L. barrettoi & - & - & - & 3 & 1 & 2 & 13 & 4 & 9 \\
\hline & B. guimaraesi & - & - & - & - & - & - & 15 & 5 & 10 \\
\hline & *o utras & - & - & - & 3 & 2 & 1 & 23 & 16 & 7 \\
\hline & Total & 62 & 22 & 40 & 292 & 135 & 157 & 205 & 128 & 77 \\
\hline & Horas Gastas & 144 & 144 & 144 & & & & & & \\
\hline \multirow[t]{8}{*}{$100 \mathrm{~m}$} & L. intermedia & 7,2 & 1,5 & 5,6 & 107,9 & 50,1 & 57,8 & - & - & - \\
\hline & L. migonei & 3,5 & 1,2 & 2,2 & 42,0 & 14,8 & 27,1 & 0,0 & - & 0,0 \\
\hline & L. fischeri & 3,4 & 3,4 & - & 13,4 & 13,4 & - & 4,4 & 4,4 & - \\
\hline & L. edwardsi & - & - & - & 0,3 & 0,1 & 0,2 & 1,1 & 0,9 & 0,2 \\
\hline & L. firmatoi & - & - & - & - & - & - & 1,3 & 0,9 & 0,3 \\
\hline & L. schreiberi & - & - & - & 0,0 & 0,0 & - & 1,0 & 0,4 & 0,6 \\
\hline & *O utras & - & - & - & 0,4 & 0,3 & 0,0 & 1,1 & 0,5 & 0,6 \\
\hline & Total & 14,1 & 6,2 & 7,9 & 164,3 & 79,0 & 85,2 & 9,2 & 7,2 & 1,9 \\
\hline \multirow[t]{10}{*}{$300 m$} & L. migonei & 2,4 & 0,4 & 2,0 & 10,2 & 3,5 & 6,6 & 0,2 & 0,1 & 0,0 \\
\hline & L. longipalpis & 1,1 & 0,3 & 0,7 & 6,2 & 2,5 & 3,7 & - & - & - \\
\hline & L. firmatoi & - & - & - & 0,4 & 0,2 & 0,1 & 4,7 & 3,9 & 0,8 \\
\hline & L. edwardsi & - & - & - & 0,2 & 0,2 & - & 4,0 & 1,4 & 2,6 \\
\hline & L. fischeri & 0,7 & 0,7 & - & 2,1 & 2,0 & 0,1 & 1,0 & 1,0 & - \\
\hline & L. whitmani & - & - & - & 0,6 & 0,6 & - & 0,5 & 0,5 & - \\
\hline & L. barrettoi & - & - & - & 0,2 & 0,0 & 0,1 & 0,9 & 0,2 & 0,6 \\
\hline & B. guimaraesi & - & - & - & - & - & - & 1,0 & 0,3 & 0,6 \\
\hline & *O utras & - & -- & - & 0,2 & 0,1 & 0,0 & 1,5 & 1,1 & 0,4 \\
\hline & Total & 4,3 & 1,5 & 2,7 & 20,2 & 9,3 & 10,9 & 14,2 & 8,8 & 5,3 \\
\hline
\end{tabular}

T - total; F - fêmea; M - macho; B - Brumptomyia; L - Lutzomyia

*O utras espécies: 100m - B. avellari, B. cunhai, B. guimaraesi, L. pessoai, L. quinquefer, L. barrettoi, L. lanei e

L. monticola. $300 m$ - B. avellari, B. cunhai, L. pessoai, L. schreiberi, L. quinquefer, L. lanei e L. monticola.

Relacionando-se os dois níveis estudados, observa-se que $94 \%$ dos flebotomíneos foram capturados no peridomicílio e domicílio. Entre as quatro espécies mais numerosas - L. intermedia, L. migonei, L. fischeri e L. Iongipalpis - representando $96 \%$ do total, evidencia-se que, tanto no domicílio como no peridomicílio, somando-se todos os tipos de captura, L. intermedia foi predominante, seguida pelas outras três espécies. Entretanto, nas capturas em isca humana, no interior da casa, L. fische ri prevalece sobre L. intermedia e L. migonei, enquanto L. Iongipal pis apresenta número re- duzido de exemplares. No peridomicílio, L. intermedia foi mais ávida pelo sangue humano, seguida por L. fischeri e L. migonei. Excetuando-se L. fischeri, com números expressivos na floresta, L. mi gonei foi capturada em número reduzido, enquanto L. intermedia e L. Iongipalpis estiveram ausentes neste local. Assim sendo, na área estudada, L. intermedia, L. migonei e L. Iongipal pis estão adaptadas ao ambiente humano, enquanto L. fischeri, apesar de ter sido capturada em número expressivo no interior da casa, mostrando ser a espécie mais endófila, apresenta-se eclética quanto ao local 
Total e média, por $10 \mathrm{~h}$ de captura, de flebotomíneos, fêmeas e machos, capturados em armadilhas Disney, com isca roedor, instaladas no peridomicílio e no bananal, a 100 m do nível do mar, de março de 1984 a fevereiro de 1985, Itaguaí, Estado do Rio de J aneiro.

\begin{tabular}{lrrrrrrrr}
\hline & \multicolumn{4}{c}{ Peridomicílio } & \multicolumn{4}{c}{ Bananal } \\
Espécie & T & F & M & MH & T & F & M & MH \\
\hline L. firmatoi & 6 & 4 & 2 & 0,3 & 42 & 28 & 14 & 2,1 \\
L. migonei & 12 & 5 & 7 & 0,6 & 3 & 2 & 1 & 0,1 \\
L. intermedia & 3 & 2 & 1 & 0,1 & 9 & 6 & 3 & 0,4 \\
L. schreiberi & 2 & 2 & - & 0,1 & 8 & 5 & 3 & 0,4 \\
L. fischeri & 3 & 3 & - & 0,1 & - & - & - & - \\
Total & 26 & 16 & 10 & 1,3 & 62 & 41 & 21 & 3,2 \\
Horas gastas & 192 & & & & 192 & & & \\
\hline
\end{tabular}

T - total; F - fêmea; M - macho; MH - média por 10h de captura

Tabela 4

Número de flebotomíneos, fêmeas e machos, capturados em abrigos naturais, utilizando-se armadilha Damasceno, a 100m do nível do mar, de março de 1984 a fevereiro de 1985, Itaguaí, Estado do Rio de J aneiro.

\begin{tabular}{|c|c|c|c|c|c|c|c|c|c|c|}
\hline \multirow[t]{3}{*}{ Espécies } & \multirow{2}{*}{\multicolumn{2}{|c|}{ Bananeira }} & \multirow{2}{*}{\multicolumn{2}{|c|}{ Toca de Tatu }} & \multicolumn{4}{|c|}{ Árvore } & \multicolumn{2}{|c|}{ Total } \\
\hline & & & & & \multicolumn{2}{|c|}{ Oco } & \multicolumn{2}{|c|}{ Tronco } & & \\
\hline & $\mathrm{F}$ & $M$ & $\mathrm{~F}$ & $M$ & $\mathrm{~F}$ & $M$ & $\mathrm{~F}$ & $M$ & $\mathrm{~F}$ & $M$ \\
\hline B. guimaraesi & - & - & - & 47 & - & - & - & - & - & 47 \\
\hline L. barrettoi & - & - & - & 23 & - & - & - & - & - & 23 \\
\hline L. firmatoi & 1 & 14 & - & - & - & - & - & - & 1 & 14 \\
\hline L. fischeri & - & - & - & - & 14 & - & - & - & 14 & - \\
\hline L. intermedia & - & 13 & - & - & - & - & - & - & - & 13 \\
\hline L. edwardsi & - & - & - & 12 & - & - & - & - & - & 12 \\
\hline L. monticola & - & - & - & - & 3 & - & 9 & - & 12 & - \\
\hline B. avellari & - & - & - & 8 & - & - & - & - & - & 8 \\
\hline Total & 1 & 27 & - & 90 & 17 & - & 9 & - & 27 & 117 \\
\hline Horas gastas & 18 & & 18 & & 18 & & 18 & & 72 & \\
\hline
\end{tabular}

F - fêmea; M - macho; B - Brumptomyia; L - Lutzomyia

de hematofagia, pois, também na floresta, mostra-se dominante com uma população significativa. Considerando-se que a espécie tem número, de certo modo, equilibrado nos três locais e observando-se a distância da floresta para o domicílio humano, aproximadamente 300m na estação A e 450m na estação $B$, podese sugerir que esta espécie seja a de maior dispersão.

Constatou-se que L. pessoai, tida como de hábitos si lvestres, não foi encontrada na floresta e, no peridomicílio, foi capturada em número reduzido. L. whitmani, L. firmatoi, L. ed- wardsi e L. schreiberi mostraram tendência a adaptação no peridomicílio.

Observa-se que, dos exemplares obtidos nas paredes internas e externas, a 100m do nível do mar, L. fischeri está representada apenas por fêmeas. L. intermedia e L. migonei ocorrem com número maior de machos. O número total de indivíduos do sexo masculino é bem amplo nas paredes externas, todavia, nas paredes internas, é mais equilibrada a proporção entre os sexos, indicando uma ligeira predominância de fêmeas. A 300m verifica-se uma supremacia de machos, tanto nas paredes internas como 
nas externas. L. fischeri, no entanto, ao contrário de L. migonei e L. Iongipal pis aparece com grande mai oria de fêmeas. A relação macho/ fêmea varia com o ambiente e com a espécie. Algumas vezes, há considerável desproporção entre os sexos, como éo caso de L. fi scheri. Em contrapartida, com L. migonei, em todos os ambientes, os machos são maioria. Tais situações indicam que os criadouros e abrigos naturais de L. fischeri estejam mais difundidos do que os de L. migonei e L. intermedia.

Em armadilhas luminosas, a 100m do nível do mar, evidencia-se a mesma tendência observada nas capturas em isca humana e paredes, ou seja, a grande maioria dos exemplares é atraída pela armadilha instalada no peridomicílio. Dentro da casa só ocorrem as espécies mais freqüentes, com ampla vantagem de L. intermedia sobre L. migonei e L. fischeri, que indicam números aproximados. No peridomicílio, ocorreram sete espécies, sendo L. intermedia e L. migonei bem mais numerosas que $L$. fischeri. Na floresta, foram doze espécies e a predominância é de L. fischeri. A 300m do nível do mar, comprova-se um equilíbrio entre o peridomicílio e a floresta. L. migonei é superior a L. Iongi pal pise $L$ fischeri, seguindo-se L. whitmani, L. firmatoi, L. edwardsi, L. barrettoi, B. avellari e B. cunhai.

Relacionando-se os dois níveis estudados, demonstra-se que, entre as espécies mais numerosas, o peridomicílio foi o local onde mais se capturou flebotomíneos, enquanto que entre as menos numerosas, este local foi a floresta. Levando-se em consideração a ocorrência de L. intermedia, L. migonei, L. fischeri e L. Iongipal pis, verifica-se que a primeira e a quarta não ocorrem na floresta, como já havia sido constatado em isca humana; a segunda aparece, neste local, com número reduzido e a terceira mostra um certo equilíbrio entre a floresta e o domicílio. As outras doze espécies estão mais representadas na floresta.

Apenas cinco espécies são atraídas para as armadilhas modelo Disney com isca de roedor: L. firmatoi, L. migonei, L. intermedia, L. schreiberi e L. fischeri. L. firmatoi é a mais freqüente e o bananal, o local onde ocorre maior número de exemplares da espécie.

Na área de estudo, foram examinados 109 abrigos potenciais de flebotomíneos, procurando-se dividi-los em domiciliares e silvestres, como o fizera Barretto (1943). Os resultados foram negativos para os abrigos domiciliares e peridomiciliares, contrastando com outras investigações de Barretto (1943), Forattini (1954, 1960), Gomes (1986 a e b), Deane \& Deane (1957) e Sherlock (1962), quando captura- ram vários exemplares de flebotomíneos nos anexos de animais domésticos durante as horas luminosas do dia, algumas vezes, em pleno exercício hematofágico. As tocas de tatu são os locais onde mais se captura flebotomíneos e as espécies que ocorrem são: B. guimaraesi, L. barrettoi, L. edwardsi e B. avellari. Tais resultados confirmam as pesquisas realizadas por Aguiar \& Vilela (1987) no Parque Nacional da Serra dos Órgãos. L. firmatoi e L. intermedia são apanhadas no interior do bananal, pousadas nos troncos. Quanto à primeira, supõe-se que esteja se adaptando ao ambiente modificado pelo homem e sendo atraída por roedores, como é comprovado nas capturas com armadilha Disney, acredita-se que seja este o seu local de repouso. Quanto à segunda, embora o número de exemplares não seja tão significativo, deve ser levado em consideração que, em focos de leishmaniose tegumentar, especialmente na região Sudeste, a presença desse flebotomíneo está ligada à cultura da banana. L. fischeri foi capturada em oco de árvore, a aproximadamente 5m de altura; Aguiar et al. (1985 b), no Parque Nacional da Serra dos Órgãos, Estado do Rio de Janeiro, e Aguiar et al. (1989), no norte do Estado do Paraná, constataram a presença da espécie em ocos de árvores, além de evidenciar seus hábitos acrodendrófilos. Tais resultados levam a acreditar que a espécie procure estes locais para se abrigar durante as horas luminosas do dia.

Pelos resultados encontrados em Itaguaí, pode-se incriminar L. intermedia como o principal vetor potencial da Leishmania (V.) braziliensis, pois, mesmo sem a constatação de infecção natural nos exemplares dissecados, a espécie demonstra ser antropofílica e totalmente adaptada ao ambiente modificado pelo homem, além da constatação de sua prevalência em domicílios onde ocorreram casos de leishmaniose tegumentar. Sua ausência do ambiente florestal reforça a hipótese de que a transmissão ocorra no ambiente domiciliar. Quanto a L. migonei, embora não seja tão antropofílica quanto L. intermedia, pode atingir o homem e os animais domésticos pela sua adaptação ao ambiente domiciliar; assim sendo, pode estar transmitindo a Le. braziliensi s como vetor secundário. L. fischeri, pela sua antropofilia e certo grau de ecletismo quanto a hora e local de hematofagia, pode ser um coadjuvante na transmissão do agente morbígeno de leishmaniose tegumentar na área estudada. Embora tenha sido a espécie mais endófila, tem uma população significativa na floresta e é nesse local a mais prevalente, podendo estar participando da transmissão em seu ciclo enzoótico 
natural. A presença de L. Iongi pal pis em área mais seca é de grande interesse epidemiológico, pois, mesmo sem a constatação de casos de calazar durante o período da pesquisa, pode haver risco para a população local, especialmente pela proximidade da região estudada com outras onde ocorreram casos da doença, particularmente os bairros de Campo Grande e

\section{Agradecimentos}

Ao Dr. Jürgen Doberainer e à Dra. Joana Doberainer, ilustres pesquisadores da Embrapa e proprietários do sítio Porangaba, pelas instalações e facilidades concedidas ao longo do trabalho. Aos biólogos Anna de Fátima Lima Klein, Denise Barone, Karla Silva Bezerra, Pedro Schuback e Thais Soucasaux Mendes Pires, pela participação eficiente nos trabalhos de campo e laboratório. Aos empregados e caseiros do sítio Porangaba, Gilberto Maria Inácio, Irene Fernandes Inácio, Josias José Inácio e Maura dos Reis Inácio, pela cordialidade.

\section{Referências}

AGUIAR, G. M.; MEDEIROS, W. M.; SANTOS, T. G.; KLEIN, A. F. L. \& FERREIRA, V. A., 1993. Ecology of sandflies in a recent focus of cutaneous leishmaniasis in Paraty, littoral of Rio de Janeiro State (Diptera, Psychodidae, Phlebotominae). Memórias do Instituto Oswaldo Cruz, 88:339-340.

AGUIAR, G. M.; SCHUBACK, P. A.; VILELA, M. L. \& AZEVEDO, A. C. R., 1985a. Aspectos da ecologia dos flebótomos do Parque Nacional da Serra dos Órgãos, Estado do Rio de Janeiro. II - Distribuição vertical (Diptera, Psychodidae, Phlebotominae). Memórias do Instituto Oswal do Cruz, 80:187-194.

AGUIAR, G. M. \& VILELA, M. L., 1987. Aspects of the ecology of sandflies at the Serra dos Órgãos National Park, State of Rio de Janeiro. VI. Shelters and breeding places (Diptera, Psychodidae, Phlebotominae). Memórias do Instituto Oswaldo Cruz, 82:585-586.

AGUIAR, G. M.; VILELA, M. L.; FERREIRA, V. A. \& SANTOS, T. G., 1989. Ecologia dos flebótomos em um recente foco ativo de leishmaniose tegumentar no Norte do Estado do Paraná (Diptera, Psychodidae, Phlebotominae). Memórias do Instituto Oswaldo Cruz, 84:7-8.

AGUIAR, G. M.; VILELA, M. L.; FERREIRA, V. A.; SANTOS, T. G.; BARONE, D.; KLEIN, A. F. L. \& MEDEIROS, W. M., 1991. Ecologia dos flebotomíneos do Parque Estadual da Serra do Mar, Picinguaba, orla marítima da escarpa atlântica do Estado de São Paulo. I - Avaliação da freqüência intra, peri e extradomiciliar. In: Resumos do XVIII Congresso Brasileiro de Zoologia. Salvador: Universidade Federal da Bahia.
Guaratiba, subúrbios da cidade do Rio de Janeiro (Marzochi \& Marzochi, 1994). As migrações humanas para a área, com a implantação do pólo petroquímico e do complexo portuário de Sepetiba, trarão um risco potencial de veiculação da leishmaniose visceral nessa região, principalmente considerando-se a provável baixa imunidade da população.
AGUIAR, G. M.; VILELA, M. L.; SCHUBACK, P.; SOUCASAUX, T. \& AZEVEDO, A. C. R., 1985b. Aspectos da ecologia dos flebótomos do Parque Nacional da Serra dos Órgãos, Rio de Janeiro. IV. Freqüência mensal em armadilhas luminosas (Diptera, Psychodidae, Phlebotominae). Memórias do Instituto Oswaldo Cruz, 80:465-482.

ALONSO, M. T. A., 1977. Vegetação. In: Geografia do Brasil, Região Sudeste, pp. 91-118. Rio de Janeiro: Fundação Instituto Brasileiro de Geografia e Estatística.

ARAÚJO FILHO, N. A., 1978. Epidemiologia da Leishmaniose Tegumentar Americana na Il ha Grande, Rio de Janeiro. Estudo sobre a Infecção Humana, Reservatórios e Transmissores. Tese de Mestrado, Rio de Janeiro: Faculdade de Medicina, Universidade Federal do Rio de Janeiro.

BARRETTO, M. P., 1943. Observações sobre a Biologia em Condições Naturais dos Flebótomos do Estado de São Paulo (Diptera, Psychodidae). Tese de Docência Livre, São Paulo: Faculdade de Medicina, Universidade de São Paulo.

DAMASCENO, R. G., 1955. Contribuição entomológica. Descrição de um método de captura de insetos em troncos de árvores, buracos na terra e tocas de animais silvestres. Resumos do XII Congresso de Higiene. Belém.

DEANE, L. M. \& DEANE, M. P., 1957. Observações sobre abrigos e criadouros de flebótomos no Nordeste do Estado do Ceará. Revista Brasileira de Malariologia e Doenças Tropicais, 9:225-246.

DISNEY, R. H. L., 1966. A trap for Phlebotominae sandflies attracted to rats. Bulletin of Entomological Research, 56:445-451. 
FALCÃO, A. R., 1981. Um novo modelo de armadilha luminosa de sucção para pequenos insetos. Memórias do Instituto Oswaldo Cruz, 76:303-305.

FALQUETO, A.; COURA, J. R.; BARROS, G. C.; GRIMALDI FILHO, G.; SESSA, P. A.; CARIAS, V. R. D.; JESUS, A. C. DE \& ALENCAR, J. T. A. DE, 1986. Participação do cão no ciclo de transmissão da leishmaniose tegumentar no Município deViana, Estado do Espírito Santo, Brasil. Memórias do Instituto Oswaldo Cruz, 81:155-163.

FORATTINI, O. P., 1954. Algumas observações sobre a biologia de Phlebotomus (Diptera, Psychodidae) em região da bacia do Rio Paraná (Brasil). Arquivos da Faculdade de Higiene e Saúde Pública da Universidade deSão Paulo, 8:15-136.

FORATTINI, O. P., 1960. Novas observações sobre a biologia de flebótomos em condições naturais (Diptera, Psychodidae). Arquivos da Faculdade de Higiene eSaúde Pública da Universi dade de São Paulo, 25:209-215.

FORATTINI, O. P. \& OLIVEIRA, O. DE, 1957. Um foco de leishmaniose tegumentar na Zona Sul do Estado de São Paulo, Brasil. Arquivos da Faculdade de Higiene e Saúde Pública da Universidade de São Paulo, 11:23-24.

FORATTINI, O. P.; RABELLO, E. X.; SERRA, O. P.; COTRIM, M. D.; GALATI, E. A. B. \& BARATA, J. M. S., 1976. Observações sobre a transmissão de leishmaniose tegumentar no Estado de São Paulo, Brasil. Revista de Saúde Pública, 10:31-43.

FORATTINI, O. P. \& SANTOS, M. R, 1952. Nota sobre a infecção natural de Phlebotomus intermedius (Lutz e Neiva, 1912) por formas em leptomonas, em um foco de leishmaniose tegumentar americana. Arquivos da Faculdade de Higienee Saúde Pública da Universidade de São Paulo, 17:171174.

FUNDREN, 1976. Plano Diretor de Itaguaí. Rio de Janeiro: Secretaria Municipal de Itaguaí.

GOMES, A. DE C., 1986a. Mecanismos e significado epidemiológico da domiciliação. Revista de SaúdePública, 22:385-390.

GOMES, A. DE C., 1986b. American leishmaniasis epidemiology in Brazil. Insect Science and its Applications, 7:161-169.

GOMES, A. DE C. \& GALATI, E. A. B., 1989. Aspectos ecológicos da leishmaniose tegumentar americana. 7. Capacidade vetorial flebotomínica em ambiente florestal primário do sistema da Serra do Mar, região do Vale do Ribeira, Estado de São Paulo, Brasil. Revista de Saúde Pública, 23:136142.

GOMES, A. DE C.; RABELLO, E. X.; SANTOS, J. L. F. \& GALATTI, E. A. B., 1980. Aspectos ecológicos da leishmaniose tegumentar americana. 1 - Estudo experimental da freqüência de flebotomíneos a ecótopos artificiais com referência especial a Psychodopygus intermedius. Revista de Saúde Pública, 14:540-556.
GUIMARÃES, A. E.; MOTTA, M.; ARLÉ, M.; MACHADO, R. \& GONÇALVES, L. D., 1989. Bionomia de mosquitos (Diptera-Culicidae) em áreas da mata Atlântica no Município de Itaguaí, Estado do Rio de Janeiro, Brasil. I. Freqüência Intra, Peri e Extradomiciliar. Memórias do Instituto Oswaldo Cruz, 84 (Supl. 4):243-254.

MARTINS, A. V.; WILLIAMS, P. \& FALCÃO, A. L., 1978. American sandflies (Diptera, Psychodidae, Phlebotominae). Rio de Janeiro: Academia Brasileira de Ciências.

MATTOS, E. A., 1981. Bionomia dos Flebotomíneos de Perobas, Município deViana (ES), Área Endêmica de Leishmaniose Tegumentar Americana. Dissertação de Mestrado, Belo Horizonte: Universidade Federal de Minas Gerais.

MARZOCHI, M. C. A.; MARZOCHI, K. B. F. \& CARVALHO, R. W., 1994. Visceral leishmaniasis in Rio de Janeiro. Parasitology Today, 10:37-40.

MARZOCHI, M. C. A. \& MARZOCHI, K. B. F., 1994. Tegumentary and Visceral leishmaniasis in Brazil. Emerging anthroponosis and possibilities for their control. Cadernos de Saúde Pública, 10 (Supl. 2):359-375.

MAYRINK, W.; WILLIAMS, P.; COELHO, M. V.; MARTINS, A. V.; MAGALHÃES, P. A.; COSTA, C. A. DA \& FALCÃO, A. L., 1979. Epidemiology of dermal leishmaniasis in the Rio Doce Valley, State of Minas Gerais, Brazil. Annals of Tropical Medicine and Parasitology, 73:123-137.

READY, P. D.; LAINSON, R. \& SHAW, J. J., 1983. Leishmaniasis in Brazil. XX. Prevalence of enzootic rodent leishmaniasis (Leishmania mexicana amazonensis) and apparent abscence of "pian bois" (Le. braziliensis guyanensis) in plantations of introduced tree species and in other non-climax forests in Eastern Amazonia. Transactions of the Royal Society of Tropical Medicine and Hygiene, 77:775-785.

SHERLOCK, I. A., 1962. Nota sobre criadouros naturais de Phlebotomus em Salvador, Bahia (Diptera, Psychodidae). Revista Brasileira de Biologia, 22:125-127.

SOUZA, M. A. DE; SABROZA, P. C.; MARZOCHI, M. C. A.; COUTINHO, S. G. \& SOUZA, W. J. S DE, 1981. Leishmaniose visceral no Rio de Janeiro. 1 - Flebotomíneos da área de procedência de caso humano autóctone. Memórias do Instituto Oswaldo Cruz, 76:161-168.

TOLEZANO, J. E.; MACORIS, S. A. \& DINIS, J. M. P., 1980. Modificação na epidemiologia da leishmaniose tegumentar no Vale do Ribeira, Estado de São Paulo, Brasil. Revista do Instituto Adolfo Lutz, 40:49-54.

VEXENAT, J. A. \& BARRETTO, A. C., 1986a. Características epidemiológicas da leishmaniose tegumentar americana em uma região endêmica do Estado da Bahia. III. Fauna Flebotomínica. Memórias do Instituto Oswaldo Cruz, 81:293-301. 